\title{
Environment Recognition System for Biped Robot Walking Using Vision Based Sensor Fusion
}

\author{
Tae-Koo Kang, Hee-Jun Song and Gwi-Tae Park \\ Korea University \\ Korea
}

\section{Introduction}

Biped walking robots are in general composed of two open kinematical chains called legs, which are connected to a main body and two contacting points to the ground, foot. There can be additional components, but this is the basic structure to identify a biped walking robot. Since biped walking robots have to have functions of mimicking human movements such as walking, or even running, they are typically complex in design, having numerous degrees of freedom (DOF) and usually include many serial links usually over 20. Therefore, it is not easy to analyze and plan the motion by using conventional robotics theories. However, there exist control techniques capable of producing a walking motion with longterm efforts from many researches. The researches on biped walking motion planning and controlling can be largely classified into three categories, 'walking pattern generation', 'motion control' and currently being researched 'whole-body control'.

Walking pattern generation means to analyze the dynamics of a biped walking robot and plan its moving trajectory for every joint. A biped walking can be divided into two steps, double support phase, when two feet are contacted to the ground and single support phase, when one of them are in the air. Then it is possible to plan the moving trajectory for the foot and hip in single support phase by using simple function generation algorithm such as polynomial interpolation method. With the trajectories of hip and foot, trajectories for other joints, mainly knee joints, can be acquired by calculating the inverse dynamics between the joints(Craig, 1989)(Huang et al., 2001)(Shih et al., 1993). In recent years, novel trajectory generation methods using artificial intelligence algorithms such as Artificial Neural Networks and Genetic Algorithms are vigorously being researched(Kim et al., 2005)(Endo et al., 2003).

In motion control and whole body control, it is the main issue how to maintain the stability of biped walking robot while walking or doing other motions. Biped robot walking can be divided into two groups, static walking and dynamic walking, by the characteristics of walking. In the static walking motion, the motion of a robot is designed for the center of gravity (COG) on the floor never to leaves the support polygon. So the robot can stop its motion in any moment and not fall down. However, fast link motions are not possible with static walking since the dynamic couplings could affect the static equilibrium. In contrast to 
static walking, a dynamic walking is realized when the ZMP never leaves the supporting polygon(Kim et al., 2005). The concept of ZMP is considering other forces applied to a robot as well as the gravitational forces. Therefore, the motion of robot depends on the whole dynamics, and consequently this analysis makes the motion of robot more efficient, smoother and faster. The concept of whole body control is to analyze the stability of the robot considering the dynamics of not only leg parts but also other links such as waist and arms. In the aspect of whole body control, it is very important to control the robot on-line while biped walking could be realized in most cases in case of motion control of only lower parts. In recent years, there have been numerous researches on whole body control, including conventional control methods as well as novel methods such as artificial intelligence techniques(Yamaguchi et al., 1999)(Nishiwaki et al. 2004).

As mentioned above, there have been numerous achievements of biped robot walking. However, most of them have been achieved with predetermined terrain conditions, mostly flat surfaces. Therefore, it is needed to develop biped robot walking algorithms capable of walking in unknown environments and it should be researched to develop the methods of recognizing the surrounding environments of robot by using vision system or other sensors. Sensors are a necessity not only for biped walking robot but also for any moving autonomous machines. Without sensors a robot would be restricted to performing proper tasks. In biped walking robot realizations, sensors are mainly used for two purposes, checking internal states or external states of robots.

Internal states of a biped walking robot generally stand for the stability of walking and they are expressed by ZMP stability criteria in many cases. In real cases, the stability is possibly evaluated by using force sensing registers, inclinometers or gyro sensors. By utilizing those sensors, a robot can be controlled on-line to stabilize its posture using feedbacks from sensors(Kim et al., 2005)(Zheng \& Shen, 1990)(Farkas \& Asada, 2005). External states represent the surrounding environments and walking conditions of a robot. They can be obtained by using distance sensors such as ultrasonic sensors or Infrared sensors and vision cameras. Those sensors are mostly used for recognizing objects to handle them or obstacles to avoid them. Unlike wheeled robots, biped walking robots have the advantage of moving over obstacles. However, in the case of moving over an obstacle, it is critically important to attain the precise information of obstacles as much as possible since the robot should contact with the obstacle by calculating the appropriate motion trajectories to the obstacle. Unfortunately, there have been not many outcomes on this topic, dynamic motion trajectory generation. Still, the researches on biped robot walking are limited within the range of walking algorithm and stability. In addition, it is critically needed to use vision cameras to obtain precise information about surrounding environment, and the techniques of vision systems such as pinhole camera model or background subtraction, etc. do not work well with cameras on the neck of a biped walking robot since the camera consistently keeps swaying because of the high disturbances of robot walking. Therefore, most of the biped walking robots uses high-priced stereo vision system to have the depth information(Gerecke et al., 2002)(Michel et al., 2005). It is an important issue to develop efficient vision processing algorithm with a single vision camera to popularize humanoids in real world.

There still remain problems in developing biped walking robots. To progress biped walking robots to the level of humanoids, technologies in robot intelligence are in need to be more developed as well as robot motion analysis and control. The currently being developed biped walking robots including the most powerful robots at present such as ASIMO and 
HUBO, etc. have problems that they cannot be operated in unknown environments. Therefore, those robots are not possible to replace the entire human works with the current level of technology. Considering the developing speed of artificial intelligence, it seems not possible to build robots with a similar level of intelligence to the one of human beings. However, it would be possible to make a robot to mimic the behaviors of human beings by using an appropriate level of preprogrammed intelligence, communication with human beings and environment recognition system. In addition, it is extremely urgent to reduce the price of biped walking robots to an affordable level for ordinary people. It is remarkably important to build a system for recognizing surrounding environments of robot and making appropriate decisions against the change of environments. In addition, a real humanoid must have the function of self-decision making as well as executing tasks given by users.

To work or to carry out a given task in an unknown environment, a biped walking robot must recognize and react to its surrounding environments. For the purpose, a robot should detect an object and track it to deal with the object. The most common method for detecting a moving object in an image is background subtraction in the literatures of vision systems $(\mathrm{Li}$ et al., 2004). In recent years, color-based object tracking methods which use the characteristics of objects represented by color histograms have been widely used in wheeled and biped walking robot researches(Fieguth \& Terzopoulos, 1997). However, those are not appropriate methods for biped walking robots since the whole background moves with the target object when a robot walks unlike the cases of using fixed cameras. Moreover, it is needed to have the predetermined color information of target object to use color-based methods. In this chapter, a method for object detection and tracking using modified optical flow algorithm is proposed. The optical flow is a method of detecting the flow of lights between time series images and it provides the information of distribution of direction and velocities in an image(Lee, 1980). Even though, the optical flow method provides motion information, it is still not proper because all the points in images move in case of biped robot walking. To overcome this problem, a modified method of the optical flow using K-means clustering algorithm is proposed and realized. The proposed system generates the optical flow field for input images and eliminates the most common motion components considering their velocities and directions by K-means clustering, which probably belong to the background. In such way, a foreground moving object can be efficiently detected and tracked.

In addition to the object detection and tracking, the function of object recognition is very important for a biped walking robot to appropriately react to its environment conditions. In this chapter, the method of object recognition is developed to make a robot to be provided proper information to climb up and down or avoid obstacles. Unlike the tracking process of moving object, those obstacles do not move. Consequently it is not possible to detect them by the same method for moving object detection. A cascade of boosted classifier (Adaboost) is used to detect obstacles. Also, those obstacles can be categorized into a couple of groups such as walls, stairs or slopes. A hierarchical Support Vector Machines (SVM) is proposed in this chapter to classify obstacles into each category. The information from the recognition system is combined with the data obtained from other sensors to determine the current state of a robot and the total information of the obstacle so as for the robot to move around or up. The system mentioned above have been realized and verified by experiments conducted with a biped walking robot. With the system, it is expected to build a biped walking robot capable of 
efficiently moving by adapting its environment with low cost by using proposed vision system methods with a single CCD camera with sensors instead of a stereo vision system.

This chapter is organized as follows. In chapter 2, the proposed environment recognition system is introduced. More specifically, the concepts of object tracking system and obstacle recognition system for a biped walking robot are illustrated. This chapter also gives examples which utilize the proposed system. In chapter 3, the results of experiments focusing on verifying the performances of the proposed system is given. They are object tracking tests and obstacle avoiding/climbing tests using the whole proposed system. Chapter 4 concludes the paper by presenting the contributions of this paper and the recommendations for future works.

\section{Environment Recognition System}

\subsection{Overall System Structure}

The overall system is constructed as illustrated in Fig. 1. The system largely consists of two parts, a biped walking robot and a Host PC. Those are connected with a Blue-Tooth wireless communication module using RS-232C. Since the realization of a biped walking robot which autonomously moves in an unknown environment as well as executes assigned tasks by a user is the main purpose of the system, the robot is equipped with several kinds of sensors gather the information of the surrounding environments. Consequently, the robot is basically designed to move autonomously without any control from the user by recognizing the environment using sensors. For instance, the robot can control its posture stability by itself, by using gyro sensors and actuators in the waist joints.

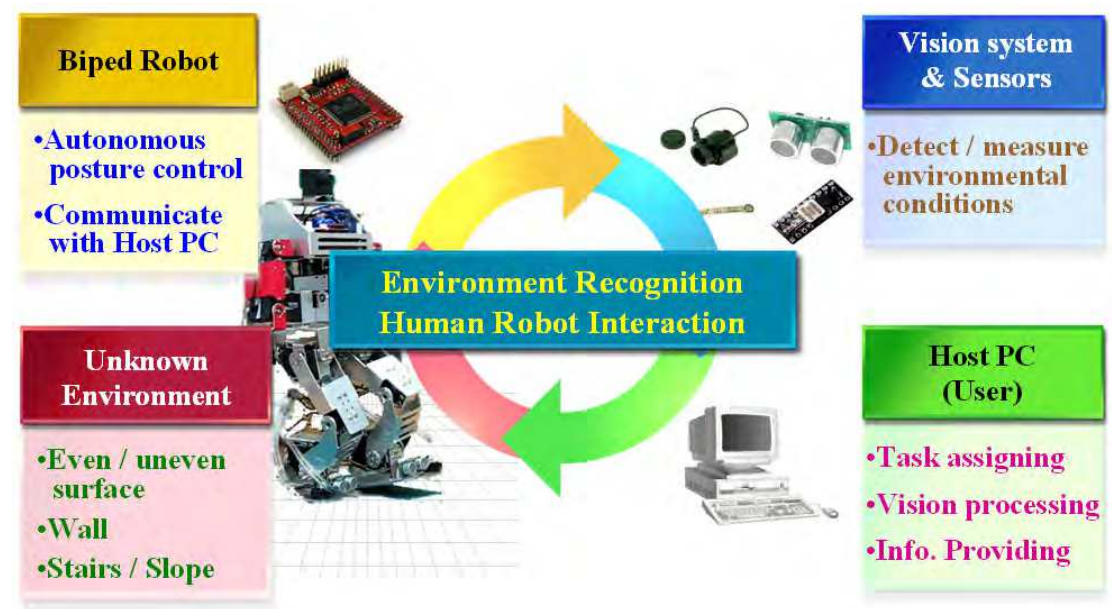

Fig. 1. Overall system architecture

In applications of biped walking robot, dynamical motion trajectory generation is more desirable than walking with a pre-determined walking motion. However, a robot must recognize its surrounding environment in advance to dynamically generate the motion trajectory. For the purpose, it is needed to develop a system of recognizing a robot's 
environment and it must be one of the fundamental conditions for more developed biped walking robot. In this chapter, environment conditions for a biped walking robot are classified into three categories: even surface, moving object and obstacles in a broad sense.

Fig. 2 shows the environment recognition system built for the biped walking robot. As shown in Fig. 2, this system is composed of two parts: moving object tracking and obstacle recognition. The moving object detecting system shown in the left of the bottom window detects and tracks a moving object by using modified optical flow method. Unlike the usual method for object detection in vision system is background subtraction, the modified optical flow method is proposed due to the always non-stationary background so that the background subtraction method detects the whole region of input image as foreground(Fieguth \& Terzopoulos, 1997)(Denavit \& Hartenbeg, 1955)(Beauchmin \& Barron, 1995). The proposed method is illustrated in more detail in chapter 2.2.

The window in the right of the bottom in Fig. 2 shows the obstacle recognition system. The possible obstacles recognized and classified with this system are obstacles which a biped walking robot can climb up and the other obstacles which it cannot climb up but should avoid. The former are again classified into stairs or slopes. For the purpose, the recognition system detects the region of an obstacle by using a cascade of boosted classifier (Adaboost) and extracts the most important features in the selected region by using Principle Component Analysis (PCA). Then the features go into a hierarchical Support Vector Machine to determine what the obstacle is. The final decision is made with other data such as distance to the obstacle from ultrasonic and infrared sensors. The more detailed descriptions are given in chapter 2.3. The environment recognition system provides not only the information of existence of objects and obstacles, but also the details of them. For instance, when the system detects a stairs in front of the robot, it estimates the distance to the stairs, the height and width of a stair so that the robot can calculate the corresponding motion trajectory to climb the stairs.

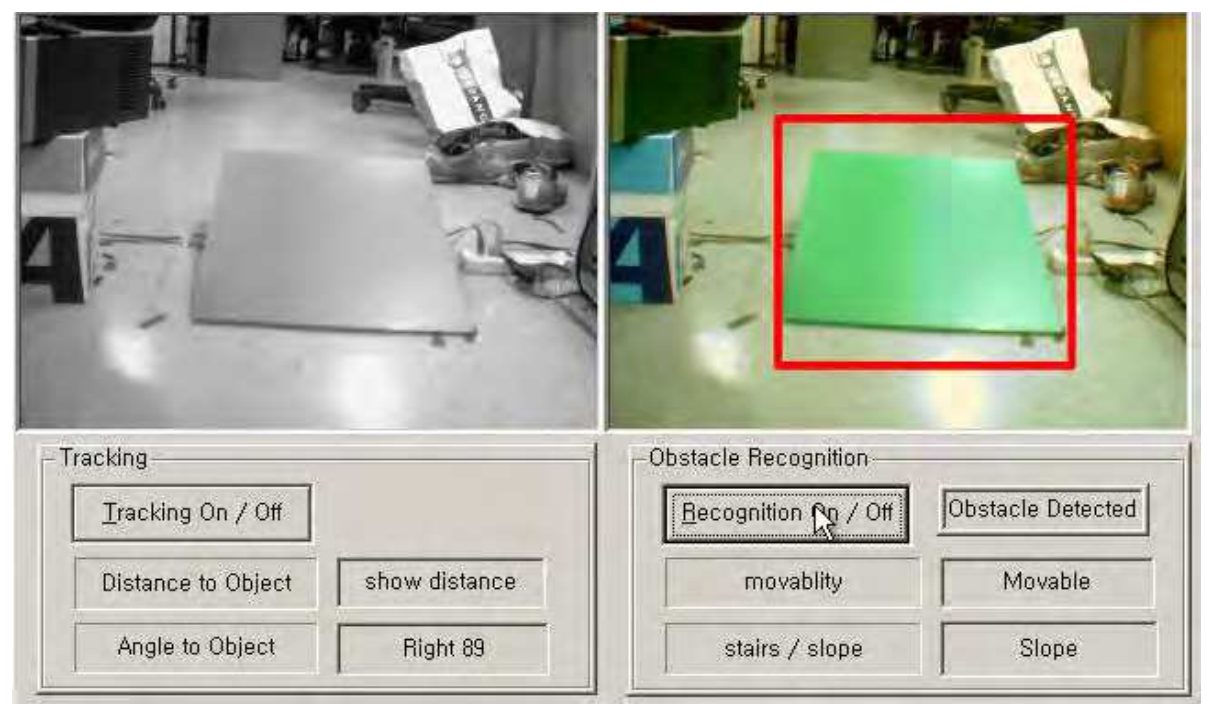

Fig. 2. Environment recognition system 


\subsection{Moving Object Detection Using Modified Optical Flow}

\subsubsection{Optical Flow Concept}

An optical flow represents the apparent velocities of movement of brightness patterns in an image. The optical flow can arise from relative motion of objects and the viewer(Denavit \& Hartenbeg, 1955)(Gibson, 1966)(Tomasi \& Kanade, 1991). It provides the information about the changes of motion between images in time. Consequently, the optical flow method has the advantages of extracting moving objects in images, no matter what the object is. How to obtain an optical flow from a series of images in time is illustrated in the following.

Let $I(x, y, t)$ be the image brightness that changes in time to provide an image sequence. Two main assumptions can be made:

- Brightness $I(x, y, t)$ smoothly depends on coordinates $(x, y)$ in greater part of the image.

- $\quad$ Brightness of every point of a moving or static object does not change in time

Let us suppose that an object moves in an image. By using Taylor series, the displacement after time $d t$ can be expressed as,

$$
I(x+d x, y+d y, t+d t)=I(x, y, t)+\frac{\partial I}{\partial x} d x+\frac{\partial I}{\partial y} d y+\frac{\partial I}{\partial t} d t+\cdots
$$

when $d t$ is small enough. Then, according to the second assumption,

$$
I(x+d x, y+d y, t+d t)=I(x, y, t)
$$

and therefore,

$$
\frac{\partial I}{\partial x} d x+\frac{\partial I}{\partial y} d y+\frac{\partial I}{\partial t} d t+\cdots=0
$$

Let us define the components of an optical flow field as,

$$
\frac{d x}{d t}=u \quad \text { and } \quad \frac{d y}{d t}=v
$$

Substituting (4) in the equation (3), the optical flow constraint equation is obtained,

$$
-\frac{\partial I}{\partial t}=\frac{\partial I}{\partial x} u+\frac{\partial I}{\partial y} v
$$

where, $u$ and $v$ are components of the optical flow field in $x$ and $y$ coordinates, respectively. Now the problem is to solve the derivative equation of (5).

Since, the vector $u$ is perpendicular to $v$, and so is $x$ to $y$, the constraint equation can be rewritten as,

$$
\left(I_{x}, I_{y}\right) \cdot(u, v)=-I_{t}
$$


where, $I_{x}, I_{y}$ and $I_{t}$ are the partial derivatives of $I$, corresponding to each subscript. Consequently, the component of an image velocity in the direction of the image intensity gradient of a point in the image is,

$$
(u, v)=\frac{-I_{t}}{\sqrt{I_{x}^{2}+I_{y}^{2}}}
$$

However, the solution to (7) cannot be directly obtained since it has more than one solution. There have been a couple of methods to solve the optical flow constraint equation by obtaining more constraints. An example of calculating an optical flow field for a rotating sphere is given in Fig. 3.

In the case of real applications, a method of feature selection based on a model of affine changes is preceded to calculating the optical flow of images, since it is computationally very expensive to apply the optical flow to every point in the images(Shi \& Tomasi, 1994).

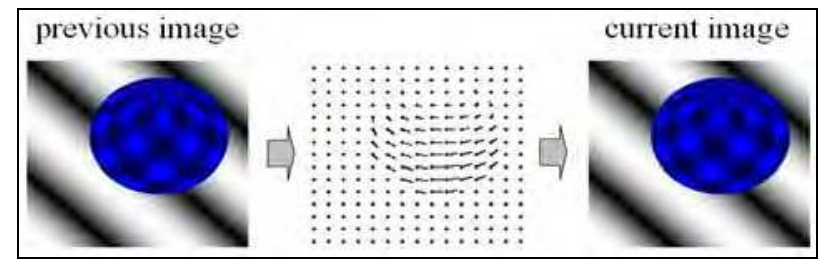

Fig. 3. Optical flow field

\subsubsection{Modified Optical Flow for Non-stationary Background}

The optical flow is an efficient algorithm for extracting moving components or tracking moving objects in images. However, the original optical flow method is not applicable in case of biped walking robot. Because the whole image varies so that all the points in an image have the optical flow components of velocities and directions. Therefore, moving objects as well as background are considered that they are moving in such case. To resolve this problem, a modification of the optical flow method is proposed to eliminate background components by using K-means clustering.

Fig. 4 represents the generated optical flow fields with a fixed camera and a moving camera, respectively. Even in case of a fixed camera, there are some extracted features to be tracked. However, it can be empirically known that the components of a moving object and ones of background are possibly classified into different groups. Therefore, in case that an object is moving in an image, the whole set of extracted motion features from the optical flow field can be classified into two groups. However, a problem still remains, that is, how to determine which group consists of the object. To overcome this problem, it is assumed that the features in a moving object have a larger distribution than ones in the background.

This might be a sound assumption since a moving object usually has various components as seen in Fig. 4, while the motion of background is almost only translational in a short time. To divide the features of each group, K-means algorithm is applied to the set in the feature space(MacQueen, 1967)(Gonzalez \& Woods, 2002). In addition, it would not be necessary to divide the object and the background in case of non-moving object. Therefore, the distance 
between the centers belonging to each group is calculated and it is considered as one group when the distance is small enough.

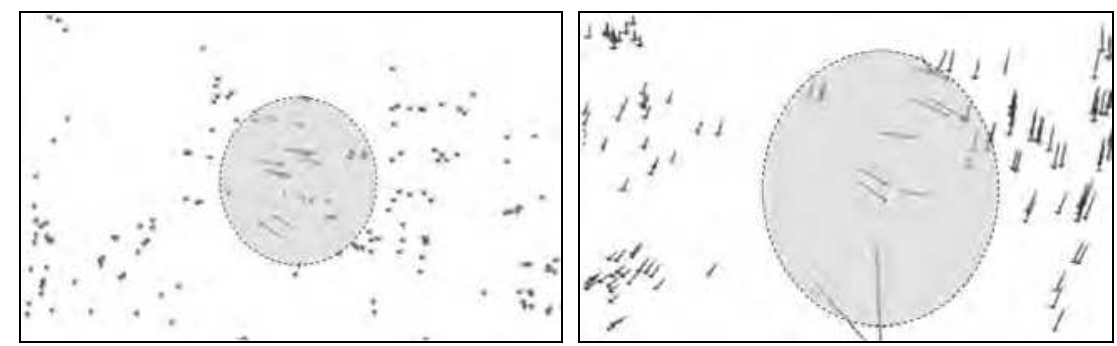

Fig. 4. Optical flow field with a fixed camera (left) and a moving camera(right)

\subsubsection{Moving object detecting system}

The detailed description of the procedure is given in the following:

1. Extract the good features to track.

2. Calculate the optical flow field and the set of components.

$$
S=\left\{u_{k}, v_{k}\right\} \quad k=1,2, \ldots, n
$$

3. For the selected components, generate a feature space denoted as $S^{\prime}$, consisting of the components of velocities and directions.

$$
S=\left\{u_{k}, v_{k}\right\} \quad \Rightarrow \quad S^{\prime}=\left\{r_{k}, \theta_{k}\right\}
$$

4. Divide the set into two clusters by using K-means clustering.

$$
\begin{aligned}
& S^{\prime}=\left\{S_{1}^{\prime}, S_{2}^{\prime}\right\}=\left\{\left(r_{l}, \theta_{l}\right),\left(r_{m}, \theta_{m}\right)\right\} \\
& \text { where } l=1,2, \ldots, n_{1}, m=1,2, \ldots, n_{2} \text { and } n=n_{1}+n_{2}
\end{aligned}
$$

5. Calculate the standard deviation of each cluster.

$$
\sigma_{k}=\sqrt{E\left(\left(S_{k}^{\prime}-E\left(S_{k}^{\prime}\right)\right)^{2}\right)}=\sqrt{E\left(S_{k}^{\prime 2}\right)-\left(E\left(S_{k}^{\prime}\right)\right)^{2}} \quad k=1,2
$$

6. Eliminate the features in the background.

$$
\begin{aligned}
& k=\max \left(\sigma_{1}, \sigma_{2}\right) \quad k=1,2 \\
& S^{\prime}=\left\{S_{1}^{\prime}, S_{2}^{\prime}\right\} \quad \Rightarrow \quad S^{\prime}=\left\{S_{k}^{\prime}\right\}
\end{aligned}
$$

7. Determine if a moving object actually exist in the image by calculating the distance between the centers of each cluster. If it is smaller than a threshold value, consider the two clusters as one cluster.

8. For the features in the selected cluster, calculate the center of gravity. It represents the center of the moving object.

$$
\begin{aligned}
& S^{\prime} \Rightarrow S(x, y, t) \\
& R=\frac{1}{N} \sum_{1}^{n} S(x, y) \quad n: \text { number of selected features }
\end{aligned}
$$


By using the proposed method, moving objects in images can be detected and tracked in most cases. Fig. 5 illustrates the whole procedure of the object tracking system.

Optical Flow field

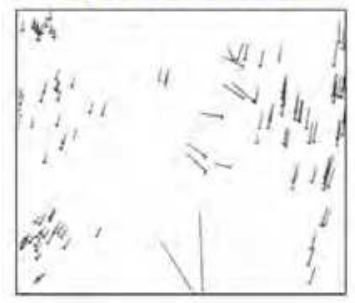

Standard deviation

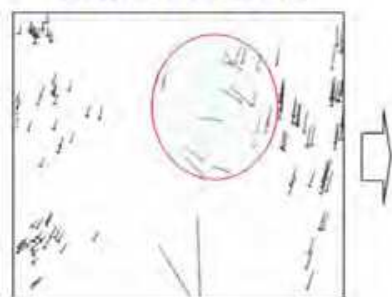

Feature space generation

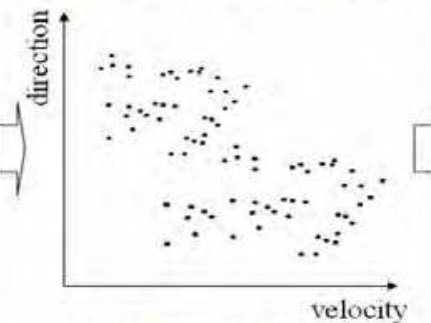

Background elimination

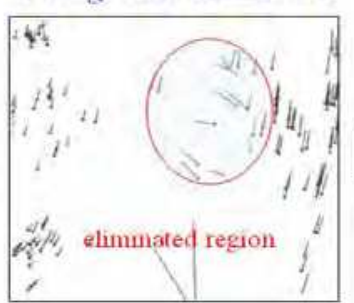

K-means clustering

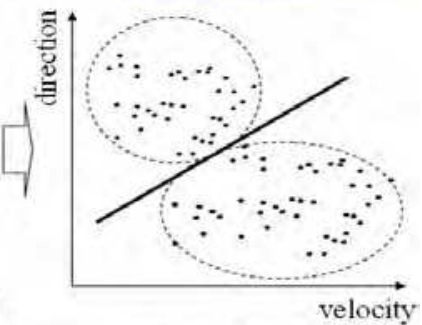

Object detection

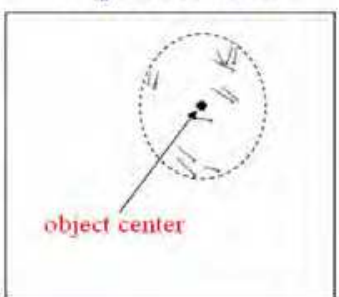

Fig. 5. Moving object detecting system

\subsection{Obstacle Recognition Using Hierarchical Support Vector Machines}

\subsubsection{Proposed Obstacle Recognition System}

The whole procedure of obstacle recognition system is illustrated in Fig. 6. As stated in previous chapters in chapter 2.1, the obstacle recognition system classifies an obstacle which a robot faces while walking and determines the details of the obstacle so that the robot is enabled to autonomously determine its behavior and generate the appropriate trajectory. This must be a mandatory system to realize humanoid robots since the current walking robots are only possible to walk in pre-programmed known environments.

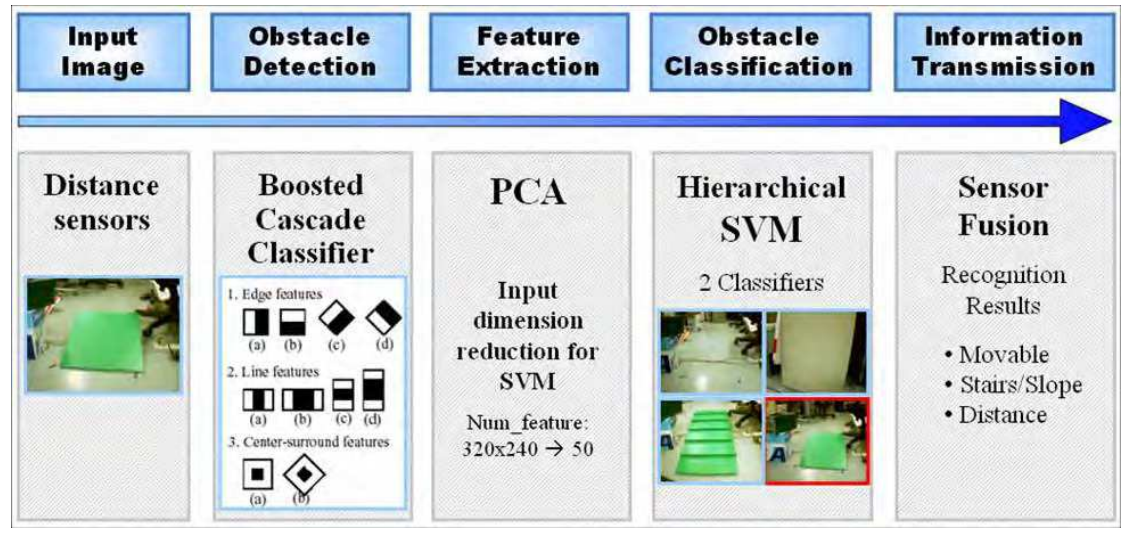

Fig. 6. Obstacle recognition system 


\subsubsection{Obstacle Detection : A Cascade of Boosted Classifier}

To recognize an object in an image obtained from the camera of a biped walking robot, it is needed to use an algorithm capable of functioning a classifier. In this chapter, a hierarchical Support Vector Machine is applied for classifying objects. However, the image ROI (region of interest) specified by the SVM classifier generally does not correctly match to the input images, there is a need of using an algorithm which sets the image ROI for the classifier. In this chapter, those algorithms of recognition using a cascade of boosted classifier and classification using $a$ hierarchical SVM are used to detect(recognize) and classify obstacles in front of a biped walking robot. For the purpose, a cascade of boosted classifier is applied to input images to specify the ROI in advance to the SVM classifier(Viola \& Jones, 2001)(Lienhart \& Maydt, 2002).

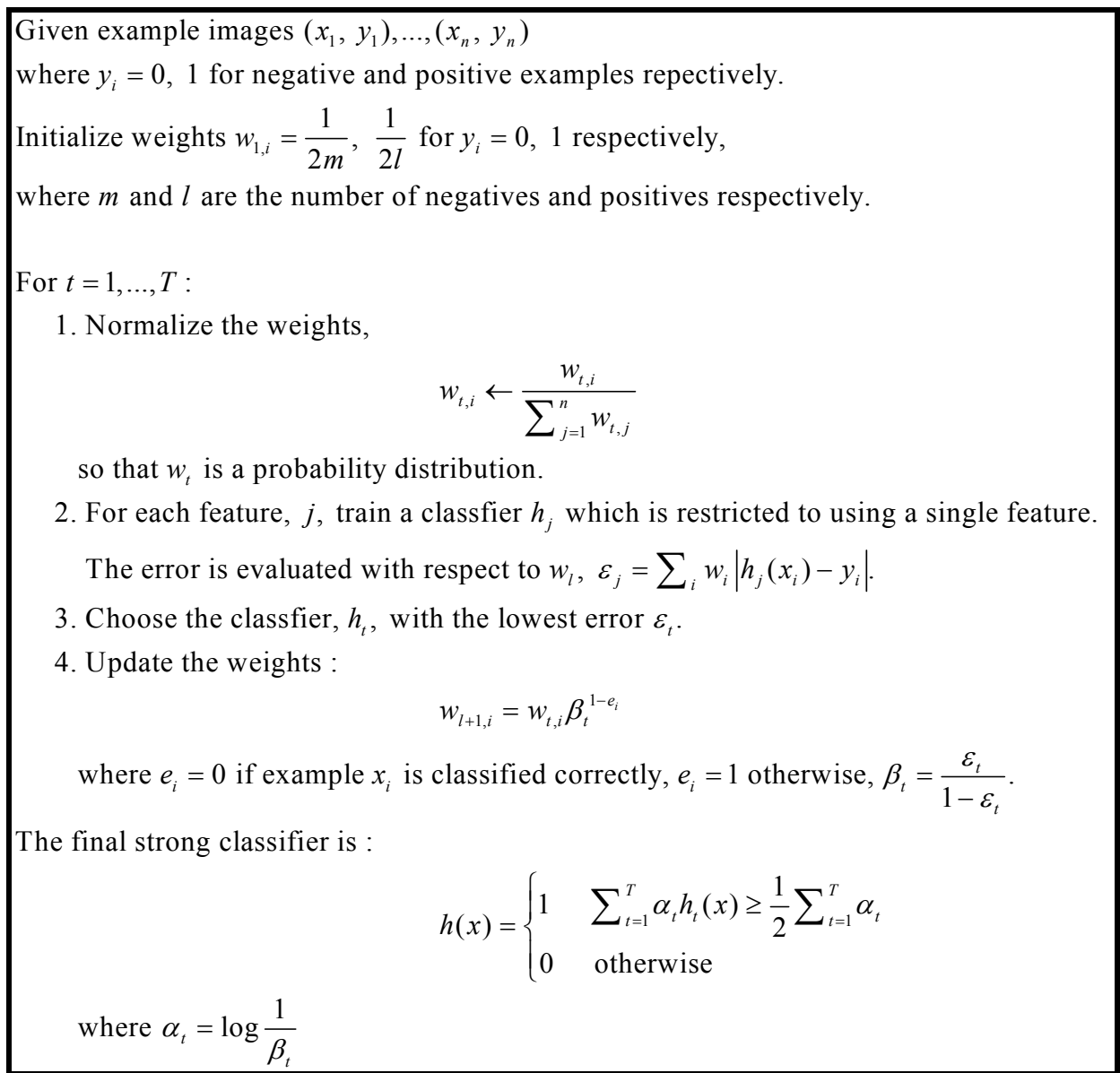

Fig. 7. Adaboost algorithm

The basic scheme of a cascade of boosted classifier, as known as Adaboost or general boosting algorithm, is to combine multiple weak classifiers into a more powerful decision 
rule for classification(Schapire, 1999). The boosted classifier is realized with a number of simple Haar filters by applying Adaboost algorithm to make the weak classifier consisting of those Haar filters to be strong. The brief algorithm of Adaboost is introduced in Fig. 7.

The algorithm performs a sequence of training rounds, and at each round a new classifier is trained. Initially, each training vector has an associated weight that encodes its importance in the learning algorithm. The training set is classified according to the decision rule of the current step, and then the weights are modified according to the classification results. This process is repeated building each time classifiers more focused on the most difficult examples. The result is a set of classifiers which combined achieve higher classification ratios.

In the boosted classifier used in the proposed obstacle detecting system, a set of Haar filters as features are used to train the classifiers. A weak classifier is not capable of detecting a rotated or translated input image. However, once a boosted classifier is generated, it is able to adaptively detect the obstacles even when they are rotated or translated. The concept of a boosted classifier and the boosted classifier used for detecting a stairs are given in Fig. 8 .

Consequently, the ROI from input image is obtained as a result of using the generated cascade of boosted classifier and a feature extraction process is performed to reduce the dimensionality of input images in advance to applying the ROI to the main classifier, a hierarchical SVM.
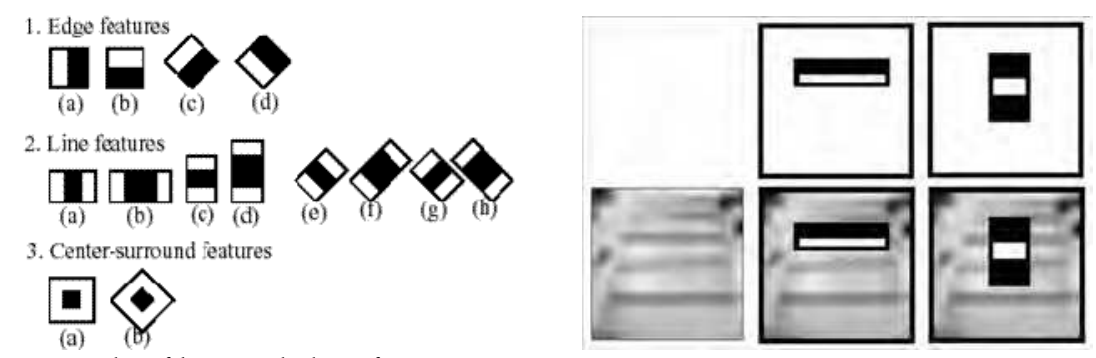

Fig. 8. A cascade of boosted classifier

\subsubsection{Feature Extraction : Principle Component Analysis}

Principle Component Analysis (PCA) is known as a useful technique to extract dominant features or reduce the dimensionality of large data sets in image processing and data mining and can also be used to find signals in noisy data in signal processing. In some cases, the dimension of the input is too large, but the components in the input are highly correlated (redundant), PCA is useful to reduce the dimension of the input. PCA has three representative effects: (1) it orthogonalizes the components of the input vectors so that they are uncorrelated with each other, (2) it orders the resulting orthogonal components (principal components) so that those with the largest variation come first, and (3) it eliminates those components that contribute the least to the variation in the data set. Since the results derived from PCA are orthogonal to each other, there is much less redundancies in the resulting data(Jolliffe, 1986).

As shown in Fig. 9, two input dataset having different dimension, 50 and 25 are generated to be modeled by SVM. First, the whole input data $(320 \times 240)$ from input images are 
transformed by PCA. Then the generated arrays having the trends of the original data (number of samples $x 50$ or number of samples $x 25$ ) are extracted. Hence, the finally resulting arrays contain 50 or 25 dimensional data containing the principal information of the original input image. These arrays are split into training and test dataset, which used to train and test using SVM.

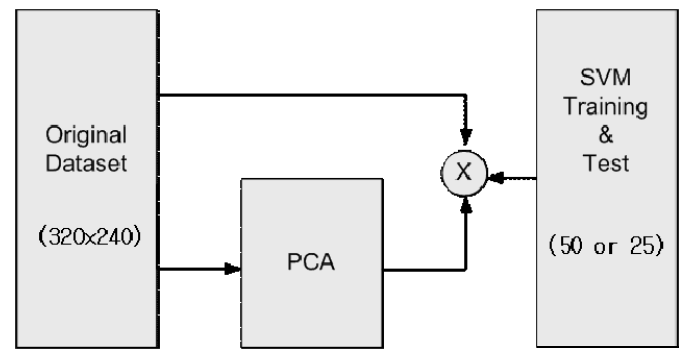

Fig. 9. Principle Component Analysis

\subsubsection{Obstacle Recognition : Hierarchical Support Vector Machines}

To recognize and classify the obstacles which a biped walking robot faces while walking, a hierarchical Support Vector Machine (SVM) is implemented to construct an efficient classifier. SVM is a learning tool originated in modern statistical learning theory. SVM can be used for a variety of applications and it is considered as a powerful classifier in the field of vision processing. The formulation of SVM learning is based on the principle of structural risk minimization. Instead of minimizing an objective function based on the training samples, such as mean square error (MSE), the SVM attempts to minimize a bound on the generalization error (i.e., the error made by the learning machine on test data not used during training). As a result, an SVM tends to perform well when applied to data outside the training set. Indeed, it has been reported that SVM-based approaches are able to significantly outperform competing methods in many applications(Burges, 1998)(Muller et al., 2001)(Wernick, 1991). SVM achieves this advantage by focusing on the training examples that are most difficult to classify. These "borderline" training examples are called support vectors(Vapnik, 1995)(Cortes \& Vapnik, 1995). The procedure how SVM classifies features in a feature space is described in the following.

Given a set of linear separating training samples $\left(x_{i}, y_{i}\right)_{1 \leq i \leq N}, x_{i} \in R^{d}, y_{i} \in\{-1,1\}$ is the class label which $x_{i}$ belongs to. The general form of linear classification function is $g(x)=w \cdot x+b$ which corresponds to a separating hyperplane $w \cdot x+b=0$. It is possible to normalize $g(x)$ to satisfy $|g(x)| \geq 1$ for all $x_{i}$, so that the distance from the closest point to the hyperplane is $1 /\|w\|$. Among the separating hyperplanes, the one for which the distance to the closest point is maximal and that is called optimal separating hyperplane. Since the distance to the closest point is $1 /\|w\|$, finding the optimal separating hyperplane amounts to minimizing $\|w\|$ and the objective function is, 


$$
\min \phi(w)=\frac{1}{2}\|w\|^{2}=\frac{1}{2}(w \cdot w)
$$

Subject to :

$$
y_{i}\left(w \cdot x_{i}+b\right) \geq 1, \quad i=1, \ldots, N
$$

If the $\mathrm{N}$ non-negative Lagrangian multipliers associated with constraints in (16) are denoted with $\left(\alpha_{1}, \cdots, \alpha_{N}\right)$, the optimal separating hyperplane can be constructed by solving a constrained quadratic programming problem. The solution $w$ has an expansion $w=\sum_{i} \alpha_{i} y_{i} x_{i}$ in terms of a subset of training patterns, support vectors. Support vectors lie on the margin. Thus, the classification function written as,

$$
f(x)=\operatorname{sign}\left(\sum_{i} \alpha_{i} y_{i} x_{i} \cdot x+b\right)
$$

When the data is not linearly separable, on the one hand, SVM introduces slack variables and a penalty factor such that the objective function can be modified as,

$$
\phi(w,)=\frac{1}{2}(w \cdot w)+C\left(\sum_{1}^{N} \zeta_{i}\right)
$$

On the other hand, the input data can be mapped through some nonlinear mapping into a high-dimensional feature space in which the optimal separating hyperplane is constructed. Thus the dot production can be represented by $k(x, y):=(\phi(x) \cdot \phi(y))$ when the kernel $\mathrm{k}$ satisfy Mercer's condition. [42] Finally, the classification function can be obtained as,

$$
f(x)=\operatorname{sign}\left(\sum_{i} \alpha_{i} y_{i} \cdot k\left(x_{i} \cdot x\right)+b\right)
$$

Because SVM can be analyzed theoretically using concepts from the statistical learning theory, it has particular advantages when applied to problems with limited training samples in the high-dimensional space. Consequently, SVM can achieve a good performance when applied to real problem. Fig. 10 illustrates the concept of SVM classification with a linear kernel.

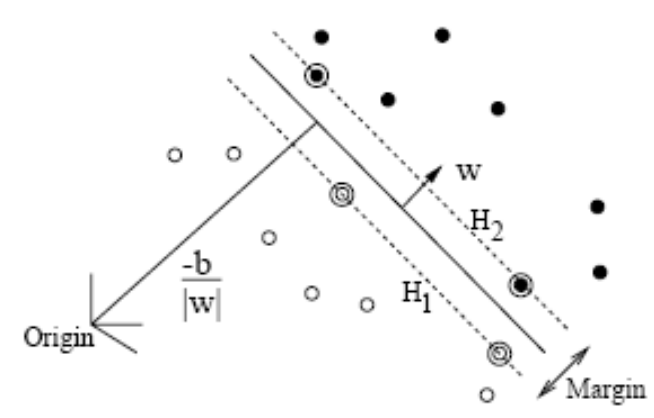

Fig. 10. SVM classification 
However, as seen in Fig. 10, the original concept of SVM is not applicable to the case of obstacle recognition for biped walking robot, because there are more than two kinds of obstacles to be classified. When a robot is walking, it could face infinite kinds of obstacles in its surrounding environments. In this chapter, obstacles are categorized largely into two groups, obstacles which a biped walking robot can climb up and ones which should be avoided. In addition, the former group can be classified into two groups again, slope and stairs, since the walking characteristics of them are quite different. Consequently, there exist at least four kinds of obstacles including an even surface. The use of the original SVM is not appropriate in this case. Therefore, in this chapter a hierarchical SVM is proposed to classify a variety of obstacles, more than two kinds.

The structure of the proposed hierarchical SVM is depicted in Fig. 11.

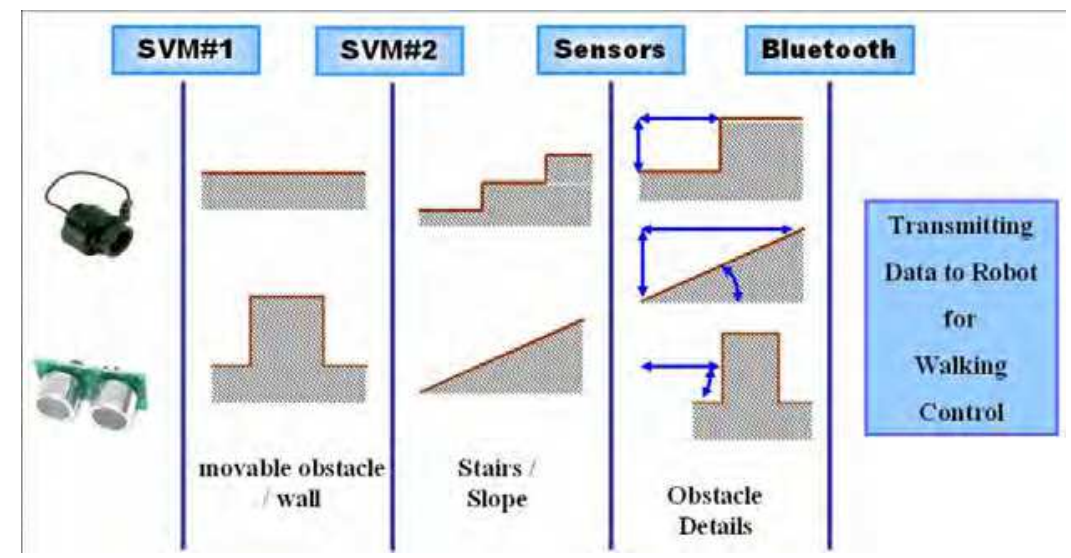

Fig. 11. Proposed hierarchical SVM structure

When an obstacle is detected by the vision camera and ultrasonic sensors installed in the robot, the input image is processed by the procedures stated in chapter 2.3.2 and 2.3.3 in advance to being applied to SVM classifier. In the classification process, a SVM classifier trained to classify even surfaces and walls is applied to the extracted features of the input image at the first stage. It determines whether the robot can climb up the obstacle or not, and returns the possibility of climbing up to the robot by using Blue-Tooth communication. Then the robot modifies its motion trajectory in a way of avoiding in case of walls or other un-climbable obstacles. If the obstacle is classified as climbable by the first SVM classifier, the features are applied to the SVM second classifier. It classifies the object into the categories of stairs or a slope. Then the recognition system determines the more detailed information such as the height and width of a stair and the inclining degree of a slope, according to the information obtained from infrared sensors. Also, the determined result is transmitted to the robot and the robot generates a corresponding trajectory to the obstacle. 


\section{Experiments and Analysis}

\subsection{Experiments for the Moving Object Detection}

\subsubsection{Experimental Conditions}

The conditions for experiments to evaluate the performance of the proposed moving object detection / tracking system is as following.

- Experiments have been largely conducted in 2 camera conditions with a fixed camera and a camera installed on the neck of the walking robot. Since the tracking system is originally designed to be robust in case that a robot does biped walking, it is expected that the performance of moving camera cases is almost similar or little lower than the one of fixed camera.

- The number of clusters in K-means clustering is set to 2. This is because it is meaningless for the walking robot to track more than 2 objects and follow them. Obviously, the tracking system can separately detect and track any number of objects by setting the correspondent number of clusters.

- The modified optical flow algorithm has 200 / 300 / 400 of features to track. As the number of tracking features becomes larger, the tracking system can be expected to be more susceptible while it might have more noises which lower the accuracy of the system.

\subsubsection{Experimental Results and Analysis}

The proposed tracking algorithm is evaluated with real-time video streams. There are 10 video streams of human moving used for the test and each video stream consists of 100 frames. Fig. 12 shows the optical flow fields in case of fixed camera and moving camera.
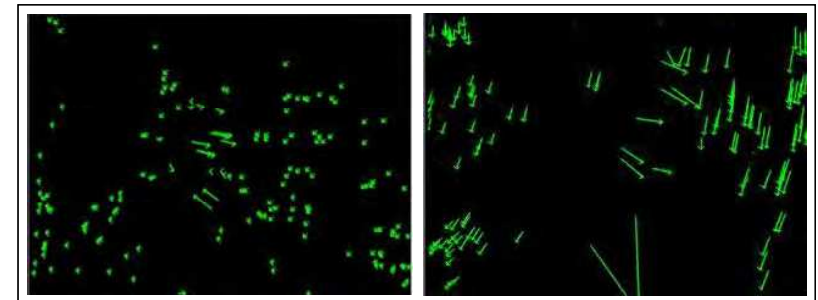

Fig. 12. Optical flow fields: fixed camera (left) and moving camera (right)

As shown in Fig. 12, the vectors in the optical flow field do not have noticeable intensities in the background region in case of a fixed camera used. In addition, it is easily seen that the number of features in the moving object region is much smaller than the one in the background region as expected. In contrast, the optical flow vectors generated by a camera mounted on the walking robot show different patterns. The vectors belonging to the background region have much larger intensities with similar directions in this case while the vectors in the object region do not have much difference from the case of fixed camera.

In both cases, the moving object regions are clearly recognized since they show remarkably different tendencies in the intensity and direction of vector elements. In the left window of Fig. 13 and Fig. 14, final results of processing the modified optical flow method are presented. The centers of moving human are marked with colored circles. 


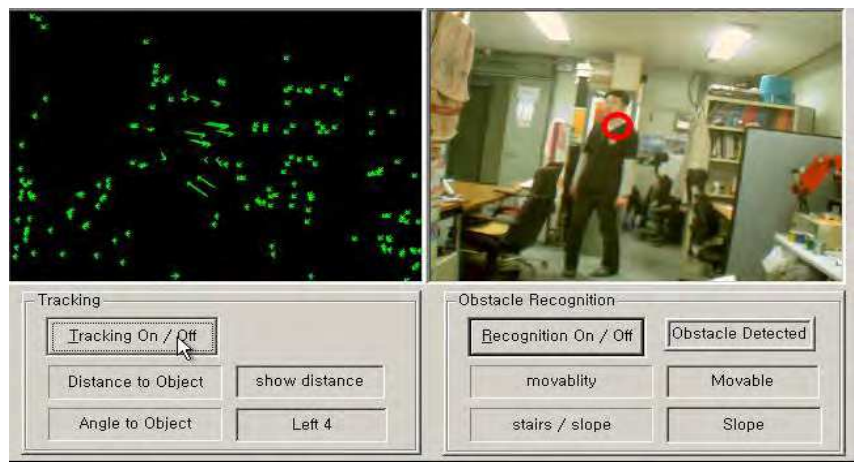

Fig. 13. Object tracking with a fixed camera

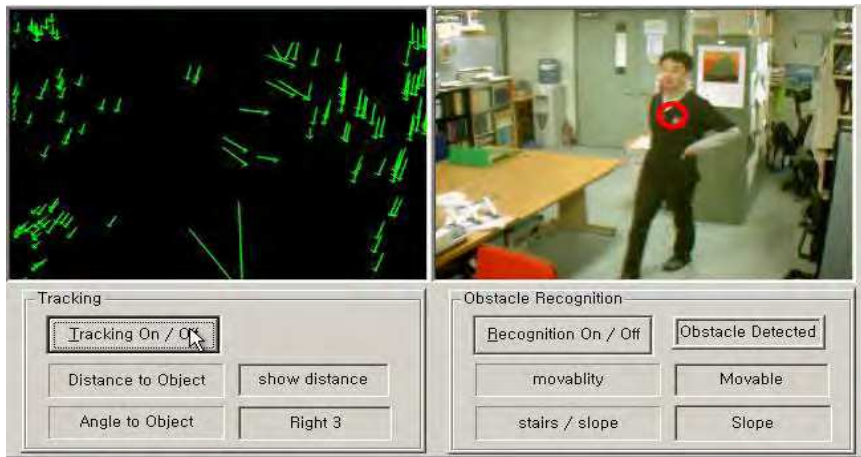

Fig. 14. Object tracking with a camera installed in a biped walking robot

From Fig. 13 and Fig. 14, it can be known that the proposed method effectively detects moving objects in both cases of fixed camera and moving camera. The evaluation of tracking accuracy is given in Table 1 . The test results indicate the number of frames which the proposed algorithm correctly detects moving object out of 1000 frames. From the results of performance tests above, it can be noticed that the proposed algorithm shows little better performance in case of fixed camera, however there is no distinguishable difference and the algorithm shows approximately $90 \%$ of accuracies in both cases.

Considering the proposed algorithm is performed in every frame so that there is no possibility of being affected by occlusions or losing objects while tracking. In addition, the average processing time is $20.9 \mathrm{~ms}$ and the camera carries out to send images at the speed of 30 frames per second. Therefore, the proposed algorithm can be concluded to be effective for moving object tracking system in biped robot walking. 


\begin{tabular}{|c|c|c|c|}
\hline camera & num_features & processing time $(\mathrm{ms})$ & tracking accuracy $(\%)$ \\
\hline \multirow{3}{*}{ fixed } & 200 & 18.2 & 91.78 \\
\cline { 2 - 4 } & 300 & 21.2 & 93.14 \\
\cline { 2 - 4 } & 400 & 23.3 & 92.52 \\
\hline \multirow{3}{*}{ moving } & 200 & 17.8 & 85.38 \\
\cline { 2 - 4 } & 300 & 21.4 & 88.43 \\
\cline { 2 - 4 } & 400 & 23.2 & 87.59 \\
\hline \multicolumn{2}{|c|}{ average } & 20.9 & 89.81 \\
\hline
\end{tabular}

Table 1. Moving object detection / tracking accuracy test results

\subsection{Experiments for the Obstacle Recognition}

\subsubsection{Experimental Conditions}

The experimental conditions for the obstacle recognition system are given in the following.

- Cascade of Boosted Classifier (Adaboost): Adaboost detects the region of interest from the input image. The output image sizes after Adaboost are set to have $25 \times 25$ pixels and 30x30 pixels. For the learning of Adaboost, 500 of wall images, 500 of slope images, 500 of stairs images and 1000 of negative images are used. Those input images are taken by rotationally and horizontally moving the obstacles. Adaboost is set to have 140 patterns (small classifiers) in 14 stages after the learning.

- $\quad$ Principle Component Analysis (PCA): PCA uses the images after Adaboost which have $25 \times 25$ or $50 \times 50$ pixels. Therefore the input data dimension to PCA is 625 or 2500 . To reduce the dimensionality of input data for hierarchical SVM, the 625 or 2500 data dimension is reduced to have 25 or 50 principle components after PCA processing.

- Hierarchical Support Vector Machine (SVM): In this stage, 2 SVM classifiers are serially connected to classify movable or not movable obstacle (wall or not) with the first classifier, then the second classifier classifies whether the movable obstacle is a slope or stairs. For the input data, the principle components having 25 or 50 dimension are used from the PCA processing. The classification accuracy is evaluated by using different kernels such as linear, polynomial and radial basis function (RBF) kernels.

\subsubsection{Experimental Results and Analysis}

The proposed obstacle recognition system is evaluated by applying 10 of 10 second video streams at the speed of 30 frames per second in each test category. (3000 frames in total) Table 2 gives the experimental result of the proposed system. The classification accuracy is measured by calculating the ratio of correct classification for the 3000 input images. 


\begin{tabular}{|c|c|c|c|c|c|c|}
\hline \multirow{2}{*}{$\begin{array}{c}\text { Adaboost } \\
\text { Ada_win_size }\end{array}$} & \multirow{2}{*}{$\begin{array}{c}\text { PCA } \\
\text { num_PC }\end{array}$} & \multirow{2}{*}{$\begin{array}{c}\text { SVM } \\
\text { SVM_kernel }\end{array}$} & \multicolumn{3}{|c|}{ accuracy (\%) } & \multirow{2}{*}{$\begin{array}{c}\text { Processing } \\
\text { time (ms) }\end{array}$} \\
\hline & & & wall & slope & stairs & \\
\hline \multirow{6}{*}{$25 \times 25$} & \multirow{3}{*}{25} & linear & 80.1 & 83.1 & 92.9 & 22.38 \\
\hline & & polynomial & 85.2 & 83.4 & 95.5 & 22.76 \\
\hline & & RBF & 88.1 & 87.2 & 97.6 & 23.21 \\
\hline & \multirow{3}{*}{50} & linear & 85.1 & 84.2 & 93.4 & 23.35 \\
\hline & & polynomial & 86.2 & 85.9 & 95.7 & 24.12 \\
\hline & & $\mathrm{RBF}$ & 87.3 & 86.2 & 97.8 & 24.06 \\
\hline \multirow{6}{*}{$30 \times 30$} & \multirow{3}{*}{25} & linear & 84.1 & 84.1 & 93.1 & 23.78 \\
\hline & & polynomial & 86.1 & 85.9 & 95.5 & 24.35 \\
\hline & & $\mathrm{RBF}$ & 87.6 & 86.6 & 97.8 & 24.89 \\
\hline & \multirow{3}{*}{50} & linear & 84.9 & 84.6 & 94.1 & 24.43 \\
\hline & & polynomial & 86.8 & 87.2 & 95.9 & 25.32 \\
\hline & & RBF & 88.4 & 86.7 & 98.1 & 25.47 \\
\hline \multicolumn{3}{|c|}{ average } & 85.83 & 85.43 & 95.62 & 22.16 \\
\hline
\end{tabular}

Table 2. Obstacle recognition performance test results

From the evaluation in Table 2, the proposed obstacle recognition algorithm shows appropriate processing time, approximately $22 \mathrm{~ms}$ and it is enough to be carried out in real time with camera at the transmission speed of 30 frames per second. The results of accuracy tests show differences by the types of obstacles. In case of wall and slope, the region detection accuracy by Adaboost is relatively high, however they also have high false alarm rate so that the total accuracies of both cases are about $85 \%$, which is not satisfactory. On the contrary, the classification accuracy of stairs is approximately $10 \%$ higher than the ones of other obstacles. In addition, cases with larger window size, larger number of principle components and RBF kernel show little better results for the cases of all types of obstacles but it is not that remarkable difference since the difference is just within $5-7 \%$.

The evaluation results can be understood that stairs have the most distinguishable features while wall and slope are similar in shape, hence the classification of wall and slope is disturbed because of the high false alarm rate caused by misclassification between wall and slope. However, this algorithm is only required to be executed every few frames since this only functions to fire the autonomous walking trajectory generation procedure of the walking robot. The proposed obstacle recognition algorithm is available to be used for biped robot walking.

Fig. 15 shows the obstacle recognition for a wall, stairs, a slope, and corresponding actual robot walking. The left window indicates the region detected by Adaboost algorithm and it is marked with a rectangle in the right window. The information window in the bottom of the right window shows the classification results. 


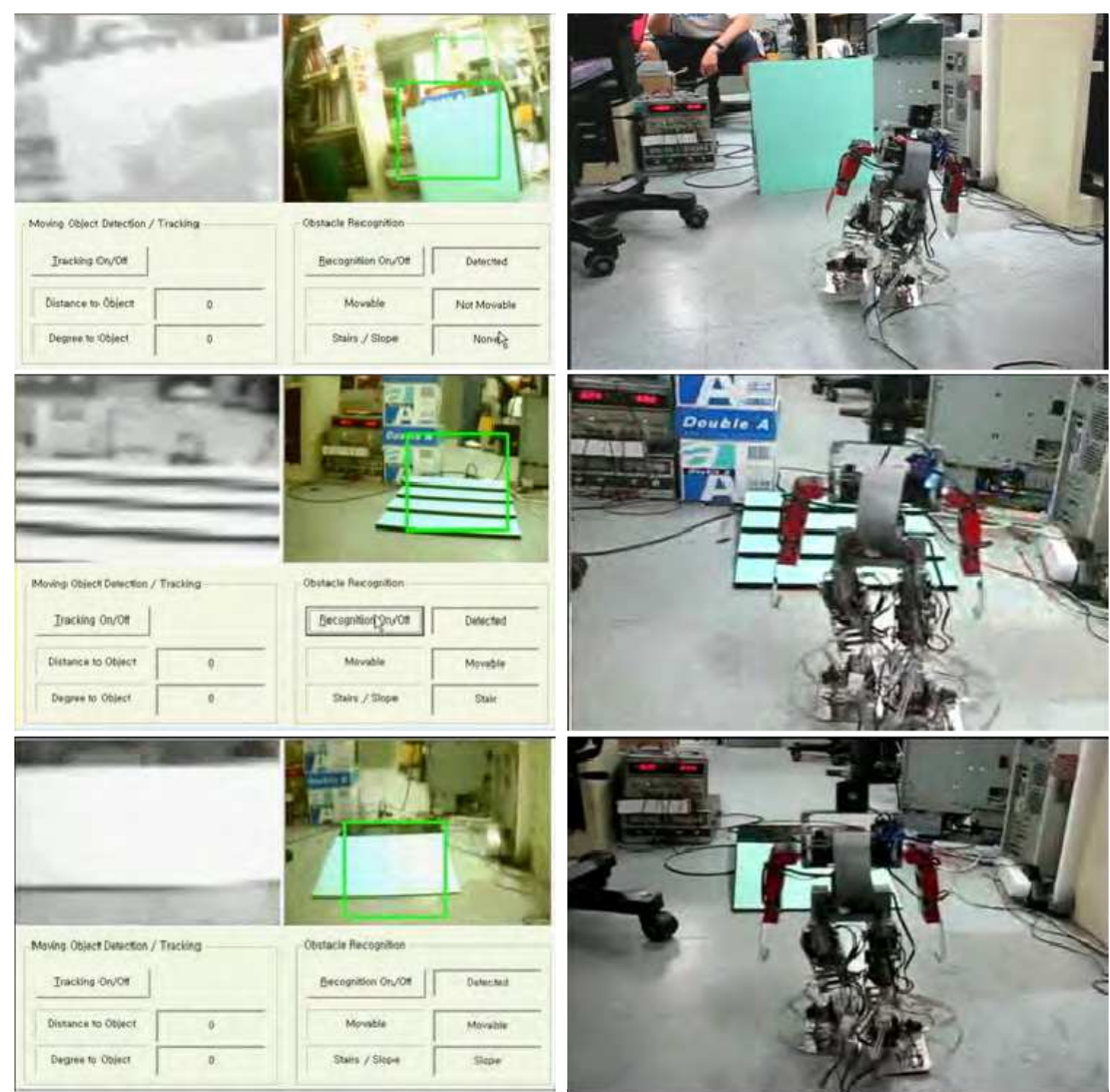

Fig. 15. Obstacle Recognition Results for a wall(top), stairs(middle), a slope(bottom)

\section{Conclusion}

This chapter presents the systems of environment recognition system and human robot interaction system for biped walking robot. For the realization of humanoid robot, they are the mandatory conditions to make a robot autonomously recognize its surrounding environment and adaptively walk by generating its motion trajectories. Therefore, this chapter has the meaning of developing aid technologies for biped robot walking control. The environment recognition system is realized by combining sensory data obtained from a walking robot including image data taken by a single camera. The problems in developing vision system in biped walking robot operated in a real world are derived from the fact that the condition for the vision system of a biped walking robot quite differs from the one of a fixed camera or a camera mounted on a wheeled robot. With a biped walking robot in real world, the commonly used vision analysis methods such as background subtraction or color-based object detection methods are not applicable since the camera always sways and it cannot be previously known what color the object has. To overcome these problems, object tracking system by using modified optical flow method and obstacle recognition system by 
using a hierarchical Support Vector Machines are proposed in this chapter. Those systems are realized and verified their effectiveness with a number of experiments by implementing them into a biped walking robot. Also, the results are presented and analyzed.

In conclusion, the systems proposed in this chapter are significantly useful in the sense that they are the characterized systems highly focused on and applicable to real-world biped walking robot. There still remain more researches to be done. They are as following:

- Because the vision system used in the proposed system totally depends on the Host PC, there is a need of developing a stand-alone vision system which works in a biped walking robot system itself.

- By using a stereo vision system, more detailed and accurate information in 3-dimension can be obtained. However, a vision system using stereo vision system costs a lot more than a single vision system in computation and money. Therefore, a way of developing more reasonable stereo vision system for biped walking robot must be developed to popularize high performance robots.

- Biped walking robot in the future should be connected to a massive network to be operated in a ubiquitous environment. Therefore, fundamental technologies for networked humanoid robot should be developed. At present, a realization of a robot connected to a TCP/IP based network such as internet could be realized.

\section{References}

Craig J.J.; (1989). Introduction to Robotics Mechanics and Control, Silma Inc..

Huang, Q., Yokoi K., Kajita S., Kaneko K., Arai H., Koyachi N. \& Tanie K. (2001). Planning walking patterns for a biped robot. IEEE Trans. on Robotics and Automation, Vol. 17, No. 3 pp.280-289.

Shih C. L., Gruver W. A. \& Lee T.T. (1993). Inverse kinematics and inverse dynamics for control of a biped walking machine, Journal of Robot Systems, Vol. 10, No. 4, pp. 531-555.

Kim D., Seo S. J. \& Park G. T. (2005). Zero-moment Point Trajectory Modeling of a Biped Walking Robot Using an Adaptive Neuro-fuzzy System, Proceedings of IEE Control Theory Appl., Vol. 152, No. 4, pp. 441-426, 2005.

Endo K., Maeno T. \& Kitano H. (2003). Co-evolution of Morphology and Walking Pattern of Biped Humanoid Robot Using Evolutionary Computation - Evolutionary Designing Method and its Evaluation, Proceedings of IEEE Intl. Conf. on Intelligent Robots and Systems, pp.340-345, Las Vegas, USA, 2003.

Yamaguchi J., Soga E., Inoue S., \& Takanishi A. (1999). Development of a Bipedal Humanoid Robot-Control Method of Whole Body Cooperative Biped Walking, Proceedings of IEEE International Conference on Robotics \& Automation, pp.368-374, 1999.

Nishiwaki K., Kuga M., Kagami S., Inaba M., \& Inoue H. (2004). Whole-body Cooperative Balanced Motion Generation for Reaching, Proceedings of IEEE Humanoid 2004, pp.672-689, 2004.

Zheng Y. \& Shen J. (1990). Gait Synthesis for the SD-2 Biped Robot to Climb Sloped Surface, IEEE Trans. Robot Automation, Vol. 6, No. 1. pp. 86-96.

Mayer N. M., Farkas F. \& Asada M. (2005). Balanced walking and rapid movements in a biped robot by using a symmetric rotor and a brake, Proceedings of Intl. Conference on Mechatronics and Automation, Vol. 1, pp. 345-350, 2005. 
Gerecke M., Albert A \& Gerth W. (2002). Vision Guided Biped Walking - Trajectories an Communication, Proceedings of the Fifth Intl. Conference on Climbing an Walking Robots and their Supporting Technologies, pp. 155-162, Paris, France, 2002.

Michel P., Chestnutt J., Kuffner J., \& Kanade T. (2005). Vision-Guided Humanoid Footstep Planning for Dynamic Environments, Proceedings of 5th IEEE-RAS Intl. Conference on Humanoid Robots, pp13-18, 2005.

Li L., Huang W., Gu I. Y. \& Tian Q. (2004). Statistical Modeling of Complex Backgrounds for Foreground Object Detection, IEEE Trans. Image Processing, Vol. 13, No. 11, pp. 1459-1472.

Fieguth P. \& Terzopoulos D. (1997). Color-based Tracking of Heads and Other Mobile Objects at Video Frame Rates, Proceedings of IEEE Conference on Computer Vision and Pattern Recognition, pp. 21-27, 1997.

Lee D. N. (1980). The Optic Flow Field: The Foundation of Vision, Philosophical Transactions of the Royal Society of London, Series B, Biological Sciences, Vol. 290, No. 1038, pp. 169-178.

Denavit J., Hartenbeg R. S. (1955). A Kinematics Notation for Lower-Pair Mechanisms Based on Matrices, ASME Journal of Applied Mechanics, pp. 215-221.

Beauchemin S. S. \& Barron J. L. (1995). The Computation of Optical Flow, ACM Computing Surveys, Vol. 27, No. 3. pp.433-467.

Gibson J. J.; (1996). The Senses Considered As Perceptual Systems, Houghton Mifflin, Boston.

Tomasi C. \& Kanade T. (1991). Detection and Tracking of Point Features, Carnegie Mellon University Technical Report CMU-CS-91-132, 1991.

Shi J. \& Tomasi C. (1994). Good Features to Track, Proceedings of IEEE Conference on Computer Vision and Pattern Recognition, pp. 593-600, 1994.

MacQueen J. B. (1967). Some Methods for classification and Analysis of Multivariate Observations, Proceedings of 5th Berkeley Symposium on Mathematical Statistics and Probability, Vol. 1, pp. 281-297, 1967.

Gonzalez R. C. \& Woods R. E. (2002). Digital Image Processing (2nd Edition), Prentice Hall.

Viola P. \& Jones M. (2001). Rapid object detection using a boosted cascade of simple features, Proceedings of IEEE Conference on Computer Vision and Pattern Recognition, Vol. 1, pp. 511-518, 2001.

Lienhart R. \& Maydt J. (2002). An Extended Set of Haar-like Features for Rapid Object Detection, Proceedings of IEEE Intl. Conference on Image Processing, Vol. 1, pp. 900-903, 2002.

Schapire R. E. (1999). A Brief Introduction to Boosting, Proceedings of Intl. Joint Conference on Artificial Intelligent, pp. 1401-1406, 1999.

Jolliffe I. T. (1986). Principal Component Analysis, Springer-Verlag, New-York, 1986.

Burges C. J. (1998). A Tutorial on Support Vector Machines for Pattern Recognition, Knowledge Discovery and Data Mining, Vol. 2, pp. 121-167.

Muller K. R., Mika S., Ratsch G., Tsuda K. \& Scholkopf B. (2001). An Introduction to KernelBased Learning Algorithms, IEEE Trans. Neural Networks, Vol. 12, pp. 181-201.

Wernick M. N. (1991). Pattern Classification by Convex Analysis, Journal of Opt. Soc. Amer. A, Vol. 12, pp. 1874-1880, 1991.

Vapnik V. (1995). The Nature of Statistical Learning Theory, Springer-Verlag, New-York.

Cortes C., \& Vapnik V. (1995). Support Vector Networks, Machine Learning, Vol. 20. pp. 273-297. 


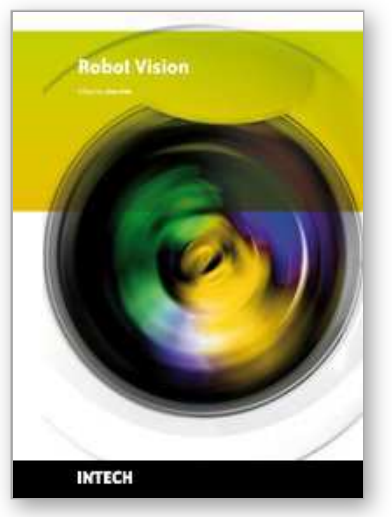

\author{
Robot Vision \\ Edited by Ales Ude
}

ISBN 978-953-307-077-3

Hard cover, 614 pages

Publisher InTech

Published online 01, March, 2010

Published in print edition March, 2010

The purpose of robot vision is to enable robots to perceive the external world in order to perform a large range of tasks such as navigation, visual servoing for object tracking and manipulation, object recognition and categorization, surveillance, and higher-level decision-making. Among different perceptual modalities, vision is arguably the most important one. It is therefore an essential building block of a cognitive robot. This book presents a snapshot of the wide variety of work in robot vision that is currently going on in different parts of the world.

\title{
How to reference
}

In order to correctly reference this scholarly work, feel free to copy and paste the following:

Tae-Koo Kang, Hee-Jun Song and Gwi-Tae Park (2010). Environment Recognition System for Biped Robot Walking Using Vision Based Sensor Fusion, Robot Vision, Ales Ude (Ed.), ISBN: 978-953-307-077-3, InTech, Available from: http://www.intechopen.com/books/robot-vision/environment-recognition-system-for-bipedrobot-walking-using-vision-based-sensor-fusion

\section{INTECH}

open science / open minds

\section{InTech Europe}

University Campus STeP Ri

Slavka Krautzeka 83/A

51000 Rijeka, Croatia

Phone: +385 (51) 770447

Fax: +385 (51) 686166

www.intechopen.com

\section{InTech China}

Unit 405, Office Block, Hotel Equatorial Shanghai

No.65, Yan An Road (West), Shanghai, 200040, China

中国上海市延安西路65号上海国际贵都大饭店办公楼405单元

Phone: +86-21-62489820

Fax: +86-21-62489821 
(C) 2010 The Author(s). Licensee IntechOpen. This chapter is distributed under the terms of the Creative Commons Attribution-NonCommercialShareAlike-3.0 License, which permits use, distribution and reproduction for non-commercial purposes, provided the original is properly cited and derivative works building on this content are distributed under the same license. 